\title{
Impaired cognitive performance under psychosocial stress in cannabis-dependent men is associated with attenuated precuneus activity
}

\author{
Weihua Zhao, PhD*; Kaeli Zimmermann, PhD*; Xinqi Zhou, MS; Feng Zhou, MS; Meina Fu, BS; \\ Christian Dernbach, PhD; Dirk Scheele, PhD; Bernd Weber, PhD; Monika Eckstein, PhD; \\ René Hurlemann, PhD; Keith M. Kendrick, PhD; Benjamin Becker, PhD
}

\begin{abstract}
Background: Deficient regulation of stress plays an important role in the escalation of substance use, addiction and relapse. Accumulating evidence suggests dysregulations in cognitive and reward-related processes and the underlying neural circuitry in cannabis dependence. However, despite the important regulatory role of the endocannabinoid system in the stress response, associations between chronic cannabis use and altered stress processing at the neural level have not been systematically examined. Methods: Against this background, the present functional MRI study examined psychosocial stress processing in cannabis-dependent men $(n=28)$ and matched controls $(n=23)$ using an established stress-induction paradigm (Montreal Imaging Stress Task) that combines computerized (adaptive) mental arithmetic challenges with social evaluative threat. Results: During psychosocial stress exposure, but not the no-stress condition, cannabis users demonstrated impaired performance relative to controls. In contrast, levels of experienced stress and cardiovascular stress responsivity did not differ from controls. Functional MRI data revealed that stress-induced performance deteriorations in cannabis users was accompanied by decreased precuneus activity and increased connectivity of this region with the superior frontal gyrus. Limitations: Only male cannabis-dependent users were examined; the generalizability in female users remains to be determined. Conclusion: Together, the present findings provide first evidence for exaggerated stress-induced cognitive performance deteriorations in cannabis users. The neural data suggest that deficient stress-related recruitment of the precuneus may be associated with the deterioration of performance at the behavioural level.
\end{abstract}

\section{Introduction}

Drug addiction has been conceptualized as a chronic and relapsing brain disorder. At the symptomatic level, the condition is characterized by escalating drug intake, progressive loss of behavioural control, withdrawal and strong craving in response to drug cues or stressors. ${ }^{1}$ Current neurobiological perspectives propose that the transition from occasional to addictive drug use is accompanied by progressive maladaptations in neural circuits engaged in reward processing, associative learning, executive control and stress reactivity. ${ }^{2}$

Cannabis is the most widely used illicit drug; $3.8 \%$ of the world's population consumes cannabis on a regular basis. ${ }^{3}$ Cannabis use-associated alterations in the domains of reward processing and cognition have been extensively studied, ${ }^{4,5}$ and there is growing evidence from functional imaging studies suggesting neuroplastic adaptations in neural systems that subserve these functions. ${ }^{6,7}$ In the cognitive domain, selective impairments in attention and working and associative memory have been reported most consistently. $4,5,8,9$

Long-term stress has detrimental effects on mental health, ${ }^{10}$ and the acute stress response is an adaptive mechanism to environmental demands that are perceived as potentially threatening. Deficient regulation of stress is a hallmark of addiction ${ }^{11,12}$ and represents a risk factor for both the escalation of drug use ${ }^{13-15}$ and relapse. ${ }^{12,16}$ Impairments in the neural circuits that mediate the acute adaptive response may contribute to reduced access to adaptive coping, so that cognitive functions, including sustained attention and regulatory control, become compromised. ${ }^{16,17}$

A growing number of functional imaging studies have reported abnormal emotional reactivity and emotion regulation

Correspondence to: B. Becker, Center for Information in Medicine, University of Electronic Science and Technology, Chengdu 611731, China; ben_becker@gmx.de

Submitted Feb. 21, 2019; Revised Apr. 15, 2019; Accepted Apr. 15, 2019; Published online Sept. 11, 2019

*These authors contributed equally to this work.

DOI: 10.1503/jpn.190039 
in cannabis users, including abnormal neural reactivity to affective stimuli ${ }^{18,19}$ and deficient amygdala downregulation during cognitive reappraisal. ${ }^{20}$ However, despite accumulating evidence for altered emotional reactivity and cognitive emotion regulation, it remains unknown whether deficient stress regulation may contribute to cannabis dependence. Support for an association between deficient stress regulation and cannabis dependence comes from large-scale surveys reporting that regulation of negative affect represents a primary motivation for cannabis use $\mathrm{e}^{21}$ and that this copingoriented motivation increases the risk of developing dependent use. ${ }^{22}$

To determine the integrity of the behavioural and neural stress response in cannabis dependence, the present study administered an established psychosocial stress induction fMRI paradigm (Montreal Imaging Stress Task; MIST) to cannabis-dependent men and matched non-using controls. During the MIST, participants perform an adaptive arithmetic task combined with negative performance feedback and critical social evaluation. To further examine the effects of stress on the urge to use cannabis, we assessed craving before and after psychosocial stress induction. Based on previous studies, we expected that cannabis-dependent participants would exhibit impaired stress-regulation capacity in the context of altered neural activity in circuits that mediate psychosocial stress processing.

\section{Methods}

\section{Participants}

Thirty-four cannabis-dependent men and 28 non-using healthy controls were recruited in cooperation with local drug counselling centres and by additional advertisements. All cannabis users fulfilled the DSM-IV criteria for cannabis dependence (Mini-International Neuropsychiatric Interview, MINI). ${ }^{23}$ To reduce variance associated with factors other than cannabis use, we decided to focus on 1 sex only and enrolled only male participants. This decision was based on previous studies reporting sex differences during psychosocial stress induction, ${ }^{24}$ stress-induced drug craving ${ }^{25}$ and menstrual cycle effects on emotion regulation. ${ }^{26}$ A similar approach has been used in previous studies on stress reactivity $^{27}$ and in studies examining emotional processing in cannabis users. . $^{19,20,28,29}$

Exclusion criteria included (1) age younger than 18 or older than 40 years; (2) left-handedness; (3) history or current DSM-IV axis I disorder (based on MINI; exception: cannabis abuse or dependence); (4) Beck Depression Inventory (BDIII) score greater than $20{ }^{30}$ (5) current or history of a medical disorder, including endocrinological abnormalities; (6) current or regular use of medication; (7) use of other illicit substances on more than 75 lifetime occasions or during the 28 days before the experiment; (8) positive urine screen for cocaine $(300 \mathrm{ng} / \mathrm{mL})$, methamphetamine $(500 \mathrm{ng} / \mathrm{mL})$, amphetamine $(500 \mathrm{ng} / \mathrm{mL})$, methadone $(300 \mathrm{ng} / \mathrm{mL})$ or opiate (300 ng/mL; Drug-Screen-Multi 7TF, von minden $\mathrm{GmbH}$, Moers, Germany); (9) breath alcohol $>0.00 \%$ (analyzed using
TM-7500, Trendmedic, Penzberg, Germany). For controls, additional exclusion criteria were applied: cumulative lifetime use of cannabis $>15 \mathrm{~g}$ (mean \pm standard deviation [SD] 1.29 \pm 1.02 ); use of any other illicit substance on more than 10 lifetime occasions. To control for confounding subacute effects of cannabis, all users were required to remain abstinent from cannabis for $24 \mathrm{~h}$ before the fMRI experiment. To increase adherence with the abstinence period, participants were informed that a urinary drug test for cannabis use would be performed on the day of the experiment. ${ }^{31,32}$

Screening procedures and fMRI assessment were scheduled on separate study days. Following an initial brief telephone screening, potential eligible participants were invited for an in-depth screening that included assessment of study participation criteria (i.e., diagnostic interview using the MINI, drug use interviews). Eligible participants were scheduled for the fMRI assessment and informed about the required abstinence time. The fMRI assessment was preceded by the assessment of potential confounding characteristics, including drug screening (i.e., urinary drug test, breath alcohol test), as well as assessment of emotional state and baseline cognitive performance indices, specifically anxiety (State Trait Anxiety Inventory; STAI) ${ }^{33}$ mood (Positive and Negative Affect Schedule; PANAS) ${ }^{34}$ and attention ( $\mathrm{d} 2$ test of attention). ${ }^{35}$ Given the high prevalence of tobacco co-use in cannabis dependence, ${ }^{36}$ the experimental groups were matched in terms of nicotine use. However, both acute nicotine administration and abstinence-induced nicotine craving may affect stress processing and underlying neural mechanisms. ${ }^{37}$ Nicotine craving is reported to peak around 3 to 6 hours after the last cigarette, and a recent study reported craving-associated neural activity changes after 4 hours of abstinence. ${ }^{38}$ As a trade-off, participants were allowed to smoke as usual, but underwent a 1.5-hour supervised abstinence period before the start of the experimental paradigm..$^{20,29}$ Following initial quality assessments, 6 cannabis users and 5 controls were excluded (Appendix 1, Figure S1, available at jpn.ca/190039-a1); the final data set included 28 dependent cannabis users and 23 healthy controls.

All participants provided written informed consent, and the study procedures had full approval by the local ethics committee. All study procedures were in accordance with the latest revision of the Declaration of Helsinki. The study was conducted at the University Hospital Bonn, Germany, from June 2015 to December 2017.

\section{Experimental design}

Psychosocial stress during fMRI acquisition was induced using the MIST. ${ }^{39}$ During the paradigm, participants were asked to perform mental arithmetic tasks and were confronted with negative feedback about their performance indicating that they performed worse than the other study participants. ${ }^{27}$ Before the experiment, participants were instructed to perform the task with high accuracy and speed. The instructions emphasized that it would be very important that participants match the average performance of the other participants and that the experimenters would 
monitor and evaluate performance online via monitor. To further increase the psychosocial stress, the experimenters criticized the participants' "bad" performance via intercom and reminded them of the importance to perform at a similar level to the other participants between the runs of the task. Participants were debriefed about the programmed failure rate after completing the study appointment. The detailed nature of the algorithm was not described, but it was emphasized that the task was designed to increase the failure rate according to their performance with the goal to induce stress.

The block-design fMRI paradigm consisted of 6 runs (each 6 minutes): 3 runs with negative feedback (stress condition) and 3 runs without feedback (no-stress control condition). The order of runs was fixed (a no-stress run was always followed by a stress run). Each run incorporated 4 blocks of 60 seconds that were preceded by a visual attention cue ( 5 seconds) and followed by a 20 second inter-block interval that served as low-level baseline (fixation cross). During the blocks, participants were required to perform an arithmetic task and to select the correct answer using a rotary dial. The participants received feedback ("correct" or "incorrect") on whether their response was correct or incorrect. During the stress blocks, additional performance indicators were displayed (own performance and average performance of the other participants). To further increase stress, a time limit was implemented that was indicated by a progressing bar moving from the left to right and "time out" was displayed in case no response occurred during the given time. An algorithm was used that adopted the response times to the performance of the participant to increase the failure rate, but this was unknown to participants. First, the average response time of the participant was determined in a pre-scan training session of 2 minutes without a time limit per arithmetic task, and the time limit for the task during fMRI was set to $90 \%$ of the participant's individual baseline response time. Furthermore, the time limit was decreased by $10 \%$ after 3 correct responses and increased by $10 \%$ after 3 incorrect responses. ${ }^{39}$

After each run, participants rated their stress level on a scale from 1 (very low) to 8 (very high). To determine baseline and stress-induced cannabis craving, all participants rated their level of cannabis craving (visual analogue scale, VAS, 0-100) before and after the paradigm. To control for between-group differences in task engagement and selfperceived performance, participants rated task enjoyment (scale of 1 to $9 ; 1=$ very unpleasant, $9=$ very pleasant) and their own performance (scale of 1 to $9 ; 1=$ very negative, $9=$ very positive) at the end of the experiment. As a physiological indicator of stress, we measured blood pressure (systolic and diastolic) at 4 different time points (at rest, 30 minutes after arrival, $\mathrm{t} 1$; immediately before the task, $\mathrm{t} 2$; immediately after the task, $\mathrm{t} 3$; 60 minutes after the task, $\mathrm{t} 4$ ). Blood pressure data for 5 participants was lost because of a technical failure, leading to a final sample size of 23 controls and 23 users for the corresponding analysis. The experiment lasted approximately 40 minutes. Stimuli were presented via liquid crystal display video goggles (Nordic NeuroLab).
Behavioural indices were analyzed using mixed analyses of variance (ANOVAs) with the between-participant factor group (control v. user) and the within-participant factor time (pre- v. post-stress; dependent variable craving; $\mathrm{t} 1 / \mathrm{t} 2 / \mathrm{t} 3 / \mathrm{t} 4$; dependent variable blood pressure) or condition (stress v. no stress; dependent variable performance or stress experience). We conducted post hoc tests to further disentangle significant main and interaction effects by comparing cannabis users and controls.

\section{fMRI acquisition and processing}

Data were acquired at $3 \mathrm{~T}$ and preprocessed using standard protocols in SPM12 (Wellcome Department of Imaging Neuroscience). The first level design matrix used a boxcar function to model the stress and no-stress conditions and also included head motion parameters as nuisance regressors (details provided in Appendix 1).

\section{fMRI BOLD-level analyses}

For analyses at the blood-oxygen-level-dependent (BOLD) level, we first investigated the stress network using a 1-sample $t$ test on the pooled data from cannabis users and controls (stress $>$ no stress). To determine altered neural stress processing in cannabis users, we conducted a 2-sample $t$ test comparing stress-related activity between groups (stress $>$ no stress). To increase the sensitivity of the analysis, the task-specific stress neural networks were defined with an independent data set from a previous study (31 healthy non-treatment male participants) using the identical paradigm. ${ }^{27}$ The fMRI data were subjected to a 1-sample $t$ test in SPM using the stress $>$ no stress contrast and thresholded at $p<0.05$ using family-wise error correction (FWE). Results revealed that the paradigm significantly engaged the middle temporal gyrus, precuneus, (para-)hippocampal gyrus and inferior parietal lobule, which were consequently considered to be stress-sensitive regions. Based on these results, bilateral anatomic masks of these 4 regions as provided in the Automatic Anatomic Labelling atlas implemented in the WFU PickAtlas ${ }^{40}$ were combined into a single mask. This mask was then used for small volume correction (SVC) using $p_{\mathrm{FWE}}<0.05$. For further post hoc analyses, parameter estimates were extracted from spheres with a $6 \mathrm{~mm}$ radius centred at the maximum $t$-value coordinates of betweengroup differences using MarsBaR. ${ }^{41}$

\section{fMRI functional connectivity analyses}

To further explore whether neural activity alterations in cannabis users were associated with altered network-level communication, a generalized form of context-dependent psychophysiological interaction (gPPI) analysis was performed. ${ }^{42}$ To this end, the task-related functional connectivity of regions that exhibited significant between-group differences in the BOLD-level analysis were examined (precuneus, see Results). The first-level gPPI models were modelled after deconvolution and included a psychological factor, a physiological factor and the interaction between the 2 factors (PPI term). In addition to the experimental conditions, head motion parameters were 
included as nuisance regressors. In line with the BOLD-level analysis, differences between cannabis users and controls were determined by means of 2-sample $t$ tests in SPM using the contrast (stress > no stress). Between-group differences in taskrelated functional connectivity were determined at the wholebrain level using $p<0.001$ uncorrected (only clusters with a minimum voxel size $>10$ reported). Given that between-group differences in task-related functional connectivity were examined using an uncorrected threshold, the corresponding findings are considered exploratory.

\section{Results}

\section{Group characteristics and drug-use patterns}

The groups did not show significant differences in several potentially confounding characteristics, including cigarette and alcohol use (Table 1). Use of other prevalent illicit drugs was low in both groups, but cannabis users reported more occasions of ecstasy use (mean $\pm \mathrm{SD}=9.72 \pm 2.19$ v. $2.33 \pm 2.31$ ).

\section{Craving, stress experience, performance and blood pressure}

Examining cannabis craving using a mixed ANOVA with group (control v. user) as a between-participant factor and time (pre- v. post-stress task) as a within-participant factor revealed significant main effects of group $\left(F_{1,49}=51.20 ; p<\right.$ $\left.0.001 ; \eta_{\mathrm{p}}{ }^{2}=0.51\right)$ and time $\left(F_{1,49}=6.23 ; p=0.02 ; \eta_{\mathrm{p}}{ }^{2}=0.11\right)$, and a significant interaction effect $\left(F_{1,49}=4.23 ; p=0.05 ; \eta_{\mathrm{p}}{ }^{2}=0.08\right)$. Post hoc analyses showed generally higher craving ratings in the cannabis group. Importantly, in the group of cannabis users, craving increased strongly after stress exposure (cannabis user group, $p=0.001$, Cohen $d=0.38$; control group, $p=$ 0.77; Fig. 1).

\begin{tabular}{|c|c|c|c|c|}
\hline Measurement $^{\star}$ & Control $(n=23)$ & User $(n=28)$ & $t\left(\chi^{2}\right)$ & $p$ value \\
\hline Age, yr & $24.57 \pm 3.55$ & $25.54 \pm 5.11$ & 0.77 & 0.45 \\
\hline Education, yr & $16.20 \pm 2.28$ & $15.95 \pm 3.68$ & 0.30 & 0.77 \\
\hline Smokers, $n$ & 19 & 26 & $1.09 \dagger$ & 0.30 \\
\hline Nicotine use, yr & $7.21 \pm 2.94$ & $9.65 \pm 5.75$ & 1.68 & 0.10 \\
\hline Cigarettes per day, $n$ & $8.45 \pm 5.34$ & $9.51 \pm 7.11$ & 0.57 & 0.57 \\
\hline Pack years & $3.04 \pm 2.44$ & $5.56 \pm 6.33$ & 1.63 & 0.11 \\
\hline Age of first alcohol use, $y r$ & $16.57 \pm 1.85$ & $15.98 \pm 3.80$ & 0.66 & 0.51 \\
\hline Days per week with alcohol use, $n$ & $1.24 \pm 1.05$ & $1.56 \pm 1.35$ & 0.90 & 0.37 \\
\hline Units of alcohol per week, $n$ & $6.31 \pm 5.67$ & $10.14 \pm 9.01$ & 1.70 & 0.10 \\
\hline Fagerström Test for Nicotine Dependence, score & $1.00 \pm 1.31$ & $1.86 \pm 2.22$ & 1.63 & 0.11 \\
\hline Age of first nicotine use, $\mathrm{yr}$ & $15.71 \pm 1.74$ & $14.96 \pm 2.49$ & 1.22 & 0.23 \\
\hline Positive and Negative Affect Schedule, positive score & $21.17 \pm 5.51$ & $20.00 \pm 6.43$ & 0.69 & 0.49 \\
\hline Positive and Negative Affect Schedule, negative score & $1.96 \pm 3.14$ & $1.29 \pm 2.75$ & 0.81 & 0.42 \\
\hline Beck Depression Inventory II score & $6.09 \pm 6.26$ & $6.50 \pm 4.42$ & 0.28 & 0.78 \\
\hline State Trait Anxiety Inventory, State score & $32.83 \pm 5.69$ & $31.71 \pm 5.77$ & 0.69 & 0.49 \\
\hline State Trait Anxiety Inventory, Trait score & $35.48 \pm 8.17$ & $35.86 \pm 7.71$ & 0.17 & 0.87 \\
\hline Age of first cannabis use, $\mathrm{yr}$ & $16.92 \pm 2.57$ & $15.68 \pm 2.82$ & 1.51 & 0.14 \\
\hline Average frequency of cannabis use (last $12 \mathrm{mo}, \mathrm{d} / \mathrm{mo}$ ) & - & $23.61 \pm 7.27$ & - & - \\
\hline Lifetime quantity of cannabis use, $\mathrm{g}$ & $1.29 \pm 1.02$ & $2309 \pm 1655$ & 7.38 & 0.001 \\
\hline Participants with past ecstasy use, $n$ & 3 & 18 & $10.71 \dagger$ & 0.001 \\
\hline Occasions of ecstasy use, $n$ & $2.33 \pm 2.31$ & $9.72 \pm 2.19$ & 2.88 & 0.01 \\
\hline Participants with past cocaine use, $n$ & 2 & 14 & $9.00 \dagger$ & 0.003 \\
\hline Occasions of cocaine use, $n$ & $5.50 \pm 4.50$ & $5.93 \pm 2.73$ & 0.08 & 0.94 \\
\hline Participants with past amphetamine use, $n$ & 4 & 18 & $8.91 \dagger$ & 0.003 \\
\hline Occasions of amphetamine use, $n$ & $3.25 \pm 4.50$ & $12.17 \pm 15.39$ & 1.13 & 0.27 \\
\hline Participants with past hallucinogen use, $n$ & - & 17 & - & - \\
\hline Occasions of hallucinogen use, $n$ & - & - & - & - \\
\hline Participants with past opiate use, $n$ & - & 3 & - & - \\
\hline Occasions of opiate use, $n$ & - & - & - & - \\
\hline Participants with past solvents use, $n$ & - & 7 & - & - \\
\hline Occasions of solvents use, $n$ & - & - & - & - \\
\hline \multicolumn{5}{|l|}{ Post-experiment ratings } \\
\hline Task enjoyment (scale of 1 to 9 ) & $6.09 \pm 1.93$ & $6.11 \pm 1.99$ & 0.04 & 0.97 \\
\hline Performance self-evaluation (scale of 1 to 9 ) & $5.87 \pm 1.33$ & $5.18 \pm 1.66$ & 1.62 & 0.11 \\
\hline
\end{tabular}


We analyzed stress experience by means of a mixed ANOVA with the between-participant factor group (control $\mathrm{v}$. user) and the within-participant factor condition (stress $\mathrm{v}$. no stress). The main effect of condition was significant $\left(F_{1,49}=\right.$ 131.91; $\left.p<0.001 ; \eta_{\mathrm{p}}{ }^{2}=0.73\right)$, indicating successful stress induction. However, we found no significant interaction effect, indicating that both groups experienced comparable levels of subjective stress.

Examining accuracy (percent correct responses) using a concordant mixed ANOVA revealed a main effect of condition $\left(F_{1,49}=60.00 ; p<0.001 ; \eta_{\mathrm{p}}^{2}=0.55\right)$, showing that both groups performed better during the no-stress condition. Furthermore, we found a main effect of group $\left(F_{1,49}=4.54\right.$; $\left.p=0.04 ; \eta_{\mathrm{p}}{ }^{2}=0.09\right)$ and a significant group $\times$ condition interaction effect $\left(F_{1,49}=5.15 ; p=0.03 ; \eta_{p}^{2}=0.10\right)$. Bonferronicorrected post hoc tests revealed that the groups exhibited

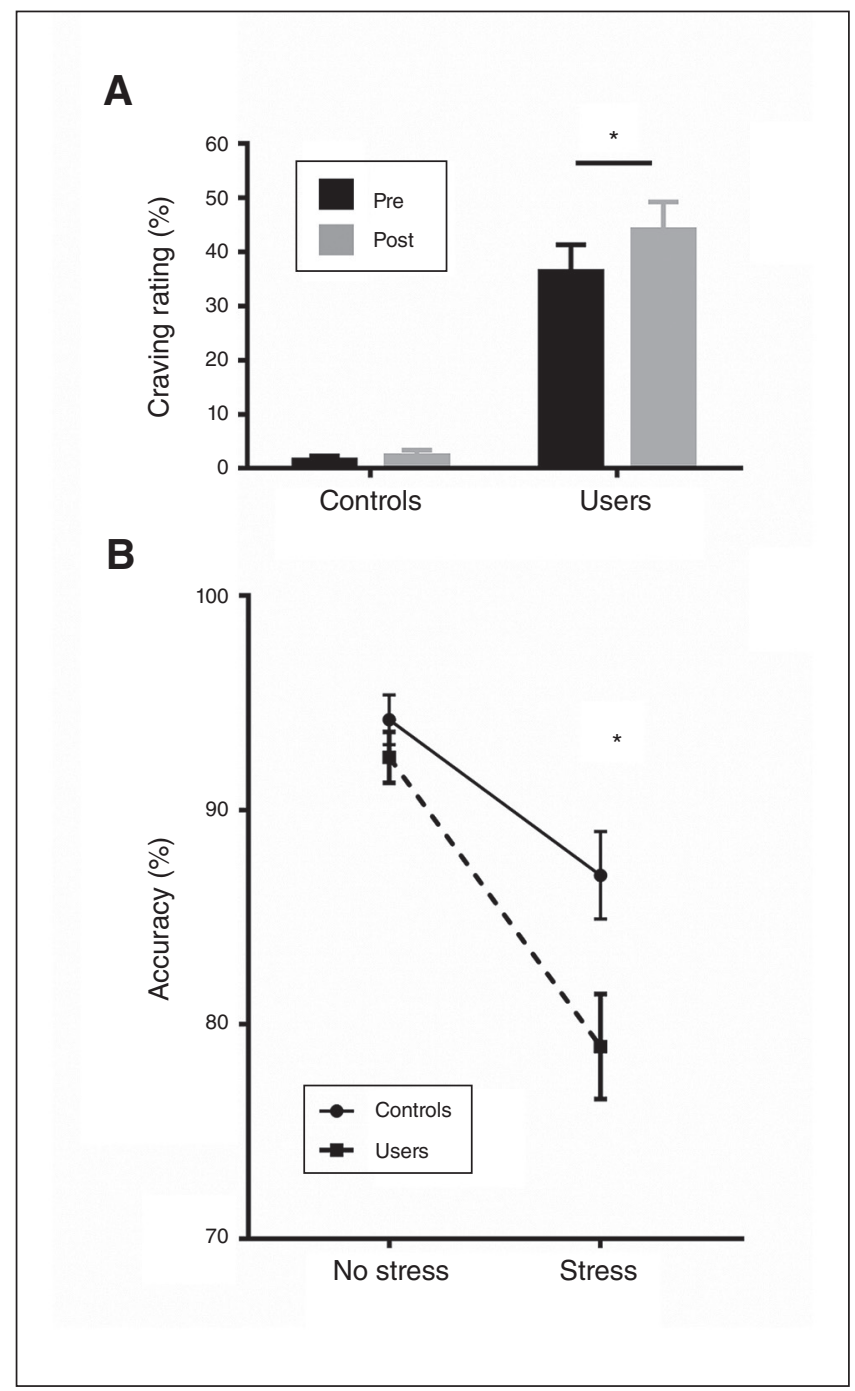

Fig. 1: (A) Cannabis craving assessed before and after stress induction. (B) Performance accuracy during the no-stress and stress conditions. Error bars reflect the standard error of the mean. ${ }^{*} p<0.05$. comparable performance during the no-stress condition $(p=0.31)$. However, under stress, cannabis users performed significantly worse than controls $(p=0.02$, Cohen $d=0.65$; Fig. 1).

Examination of blood pressure revealed a significant main effect of time $(\mathrm{t} 1 / \mathrm{t} 2 / \mathrm{t} 3 / \mathrm{t} 4)$ for systolic $\left(F_{3,132}=6.19 ; p=0.001\right.$; $\left.\eta_{\mathrm{p}}{ }^{2}=0.12\right)$ and diastolic blood pressure $\left(F_{3,132}=4.63 ; p=0.005\right.$; $\left.\eta_{\mathrm{p}}{ }^{2}=0.10\right)$. Bonferroni-corrected pair-wise comparisons illustrated that systolic blood pressure after the task was higher than 30 minutes after arrival $(p=0.03)$ and immediately before the task $(p=0.001)$, reflecting successful stress induction. Diastolic blood pressure was higher at $\mathrm{t} 3$ than at $\mathrm{t} 2(p=$ 0.006). In line with the lack of between-group differences in self-reported stress experience, both groups displayed comparable cardiovascular stress reactivity.

\section{fMRI BOLD-level analyses}

The paradigm induced widespread activity in psychosocial stress networks encompassing middle frontal regions, the precuneus and posterior cingulate cortex (Table 2; Appendix 1, Fig. S2). An examination of neural differences between the cannabis users and controls in the task-specific stress network revealed significantly decreased stress reactivity in the cannabis users compared to controls in a cluster in the right precuneus $\left(x, y, z=3,-70,50 ; k=32, p_{\mathrm{FWE}}<\right.$ 0.05; dorsal-posterior subdivision of the precuneus). Post hoc analyses of extracted parameter estimates from this region further revealed that the differences during the stress versus no-stress conditions in the precuneus were smaller in cannabis users than in controls $\left(t_{49}=2.90 ; p=\right.$ 0.006; Cohen $d=0.8$; Fig. 2) and cannabis users showed an attenuated increase during stress relative to the no-stress condition $\left(t_{27}=2.47 ; p=0.023\right.$; Cohen $d=0.32$, corresponding to a small effect size) compared to controls $\left(t_{23}=5.71\right.$; $p<0.001$; Cohen $d=0.83$, corresponding to a large effect size; Fig. 2). This was also reflected by marginally significant lower precuneus activity during stress in the cannabis users compared to controls $\left(t_{49}=1.74 ; p=0.09\right.$; Fig. 2). An exploratory whole-brain analysis revealed no significant between group differences at the whole-brain level after FWE correction.

\section{Associations between neural activity and cannabis use parameters}

In the cannabis-dependent group, we observed no significant associations between precuneus activity and cannabis use parameters (age of onset $r=-0.17, p=0.40$; cumulative lifetime use, $r=-0.24, p=0.22$; frequency of use $r=-0.15$, $p=0.44)$.

\section{fMRI functional connectivity}

An exploratory whole-brain analysis comparing stress-related connectivity of the precuneus (seed region) between cannabis users and controls revealed relatively increased connectivity of the precuneus with the superior frontal gyrus $(x, y, z=12$, 
26, 56; $k=34 ; p<0.001$, uncorrected) in the cannabis users (Fig. 3). An independent $t$ test revealed increased precuneussuperior frontal gyrus coupling in cannabis users compared with controls $\left(t_{49}=3.62 ; p=0.001\right.$; Cohen $d=1.02$, Fig. 3$)$.

\section{Discussion}

The present study examined psychosocial stress processing in cannabis-dependent males using an adaptive mental

\begin{tabular}{|c|c|c|c|c|}
\hline Hemisphere & Region & Cluster size & Peak $t$ & MNI coordinates, $x, y, z$ \\
\hline \multicolumn{5}{|c|}{ Stress $>$ no stress } \\
\hline \multirow[t]{3}{*}{ Right } & \multirow{3}{*}{$\begin{array}{l}\text { Precuneus/middle occipital } \\
\text { gyrus }\end{array}$} & \multirow[t]{3}{*}{6864} & 16.30 & $9,-76,-10$ \\
\hline & & & 16.04 & $45,-64,8$ \\
\hline & & & 15.69 & $18,-94,14$ \\
\hline \multirow[t]{3}{*}{ Right } & \multirow[t]{3}{*}{ Insula/middle frontal gyrus } & \multirow[t]{3}{*}{400} & 10.48 & $33,20,5$ \\
\hline & & & 7.73 & $33,44,20$ \\
\hline & & & 7.61 & $33,41,32$ \\
\hline \multirow[t]{3}{*}{ Right } & \multirow{3}{*}{$\begin{array}{l}\text { Middle frontal gyrus/medial } \\
\text { frontal gyrus/cingulate gyrus }\end{array}$} & \multirow[t]{3}{*}{603} & 9.72 & $39,-1,53$ \\
\hline & & & 9.14 & $45,8,32$ \\
\hline & & & 8.58 & $21,-1,59$ \\
\hline Left & Middle frontal gyrus & 69 & 7.50 & $-39,-10,50$ \\
\hline
\end{tabular}

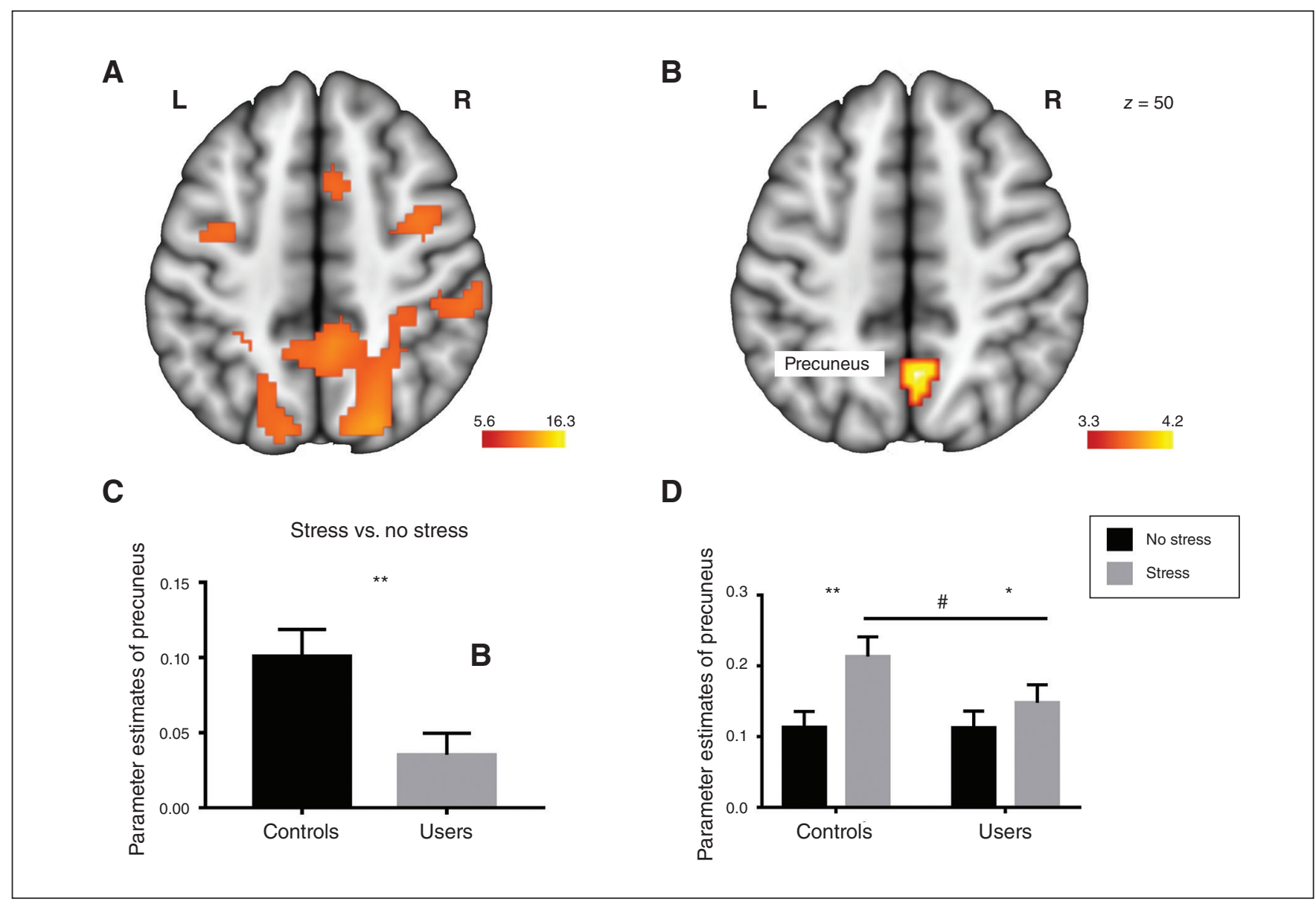

Fig. 2: Stress-related network in $(A)$ the entire sample and $(B)$ differences between cannabis users and controls, showing that cannabis users exhibited decreased precuneus activity during psychosocial stress. Extracted parameter estimates from the precuneus for the contrasts (C) stress $>$ no stress and (D) no stress v. baseline, stress v. baseline further revealed that the effect was driven by lower activity during the stress condition. Image $(\mathrm{A})$ is thresholded at $p_{\mathrm{FWE}}<0.05$. Image $(\mathrm{B})$ is thresholded at $p_{\mathrm{FWE}, \mathrm{SVC}}<0.05$. Error bars reflect the standard error of the mean. ${ }^{*} p<0.05 ;{ }^{* *} p<0.01 ; \# p=0.09$. FWE = family-wise error; $\mathrm{L}=$ left; $\mathrm{R}=$ right; $\mathrm{SVC}=$ small volume correction. 
A

Precuneus-SFG connectivity

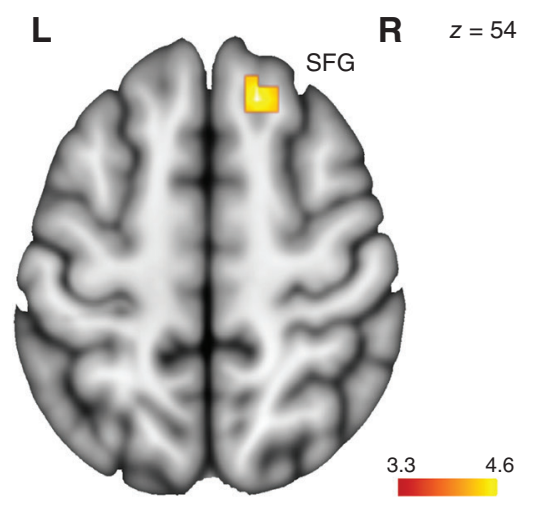

B

Stress vs. no stress

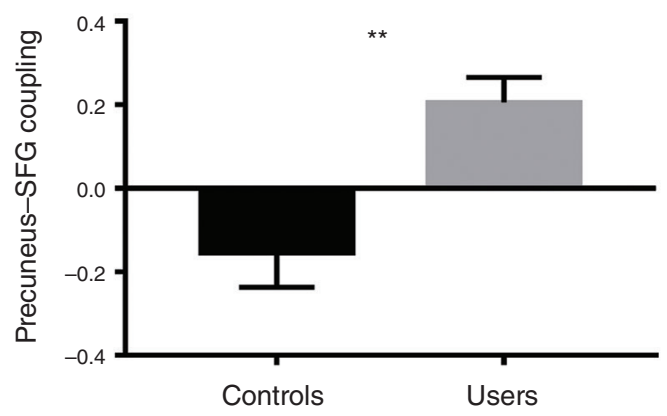

Fig. 3: Cannabis users exhibited increased functional connectivity between the precuneus (seed region) and the superior frontal gyrus during stress. (A) Location of the precuneus-superior frontal gyrus (SFG) pathway that exhibited group differences. (B) Extracted connectivity estimates from the pathway for the contrast (stress $>$ no stress). Image is thresholded at $p<0.001$, uncorrected. Error bars reflect the standard error of the mean. ${ }^{* *} p<0.01$. $L=$ left; $R=$ right.

arithmetic task accompanied by negative social evaluation. During psychosocial stress exposure, but not the no-stress condition, cannabis users demonstrated decreased performance relative to controls, despite normal stress experience and cardiovascular stress reactivity. At the neural level, stressrelated performance deteriorations in cannabis users were accompanied by decreased precuneus activity and increased connectivity of this region with superior frontal regions.

In line with previous studies, the experimental task successfully induced stress in both groups as indicated by increased subjective stress experience and cardiovascular activity. ${ }^{43}$ Although we observed no differences in cardiovascular and subjective stress reactivity, cannabis-dependent users demonstrated significantly lower arithmetic task performance during stress induction. Previous studies reported altered stress reactivity in alcohol ${ }^{44}$ and nicotine users $^{45}$ and elevated levels of anxiety and depression. ${ }^{46}$ Cannabis users and controls in our sample were comparable for these potential confounding characteristics, arguing against strong confounding effects of these factors on the observed between-group differences. Both groups exhibited high and comparable performance in the absence of stress, indicating comparable baseline cognitive performance. In line with previous studies, ${ }^{47}$ stress increased cannabis craving in dependent participants, confirming the important role of stress as a driving factor of dependence and relapse. ${ }^{15}$ Against our expectations, however, the groups did not differ with respect to the subjective stress experience (however, one study reported a normal distress experience in cannabis users during social exclusion) ${ }^{48}$ cardiovascular indices, self-perceived performance or task enjoyment. Together, these findings suggest that while stress induction and stress perception may be intact in dependent cannabis users, psychosocial stress increases cannabis craving and leads to marked deteriorations in cognitive performance.

At the neural level, lower performance in the cannabis group was accompanied by an attenuated stress-related increase in precuneus activity. The precuneus, located in the posteromedial parietal lobe, is considered to play a central role in a range of highly integrated tasks, including basic cognitive tasks (i.e., mental arithmetical performance) and social cognitive tasks, particularly self-referential and mentalizing processes, as well as cognitive effort and the impact of effort on cognitive performance. ${ }^{49-52}$ The precuneus has received little attention in neurobiological models of addiction. However, an increasing number of studies have reported altered precuneus activation in chronic cannabis users during cueinduced craving, ${ }^{53}$ as well as cognitive processing in emotional and social contexts, such as risky decision making, ${ }^{54}$ suppression of emotional distractors, ${ }^{55}$ evaluation of episodic memory episodes or mentalizing. ${ }^{56}$

Given the stronger engagement of parietal regions (including the precuneus) with increasing difficulty of mental arithmetic operations, ${ }^{49}$ additional recruitment of the precuneus may have attenuated stress-related task deteriorations in the controls. In contrast, decreased task performance during stress in cannabis users may be linked to failure of compensatory precuneus recruitment. Decreased stress-related precuneus recruitment was accompanied by increased functional connectivity of this regions with the dorsal medial prefrontal cortex, specifically the superior frontal gyrus, ${ }^{57}$ involved in both mental arithmetic performance and affective and impulse control. ${ }^{49,57,58}$ Alterations were specifically located in the dorsal-posterior subdivision of the precuneus, which 
exhibits particularly strong connections with frontal regions engaged in cognitive and executive domains. ${ }^{52,59}$ Increased dorsal-posterior precuneus-frontal coupling may thus reflect a successful compensation of negative emotional experience during performance deterioration or a deficient compensatory attempt to maintain task performance. The latter interpretation is further supported by previous reports on increased neural recruitment, ${ }^{60}$ particularly of frontal regions, in the context of normal working memory performance in cannabis users. ${ }^{61,62}$

Together with the posterior cingulate, the precuneus has been suggested as a core hub of the default mode network. ${ }^{63,64}$ Activity in this network is suppressed during external attentional demands, but increases during self-referential processes. ${ }^{65}$ Previous studies have reported altered precuneus activity in response to salient drug cues ${ }^{53}$ and external cognitive demands ${ }^{66}$ in cannabis users or users of synthetic cannabinoids, respectively. Lower engagement of this region in cannabis users thus may alternatively reflect altered self-related processing during psychosocial stress in cannabis users.

The observed psychosocial stress-specific performance impairments in cannabis-dependent participants in the present study aligned with previous studies reporting impaired cognitive performance in cannabis users in the context of negative emotional ${ }^{20}$ and social information. ${ }^{67}$ Together, these findings suggest an association between chronic cannabis use and deficient integration at the intersection between emotional and cognitive processes. The effect of stress on addiction is multifactorial, and deficient stress regulation has been determined as a risk factor for the escalation of cannabis use and dependence. ${ }^{13-15}$ The retrospective nature of the present study did not allow us to disentangle predisposing factors from consequences of chronic cannabis exposure or addictionrelated maladaptations in stress regulation. Therefore, impaired performance under stress, as well as associated neural alterations, may alternatively reflect a predisposing deficit for the development of cannabis dependence or changes in stress-related cannabinoid signalling due to cannabis exposure-related adaptations. From a clinical perspective, the present findings emphasize the detrimental impact of psychosocial stress on craving and cognitive performance in cannabis-dependent individuals. As such, exposure to psychosocial stress may promote relapse, impair cognitive performance and ultimately interfere with social and occupational rehabilitation. Therapeutic approaches that aim at improving coping strategies and increase stress resilience in cannabis-dependent individuals may therefore represent a promising strategy.

\section{Limitations}

The findings of the present study need to be considered in the context of a number of limitations. First, findings were observed in limited number of male cannabis users; the robustness of the findings and generalizability to female cannabis users therefore remain to be determined in larger samples including both sexes. Second, the groups differed in occasions of ecstasy use. Although a previous study indicated that emotional dysregulations in low-dose ecstasy users were predicted by cannabis rather than ecstasy use, ${ }^{68}$ we could not rule out a potential contribution to the observed effects. Third, the group of cannabis users included a larger proportion of people with previous experience with ecstasy, as well as amphetamine and cocaine use, and we could not completely rule out the potential effects of these substances. Fourth, the sample size was based on previous studies, ${ }^{20,27}$ but a priori estimations would support stronger conclusions with respect to the observed group differences. Finally, groups were matched with respect to nicotine use, but the contribution of complex interactive effects between nicotine and cannabis could not be ruled out. Functional connectivity differences of the precuneus were determined using an exploratory analysis and need to be interpreted with caution.

\section{Conclusion}

Overall, the present study provides the first evidence for stress-induced cognitive performance deficits in cannabis users. Importantly, deficits were observed specifically for acute stress in contrast to normal performance under no stress and normal perceived stress intensity. The neural data suggest that deficient stress-related activation of the precuneus may underpin these impairments.

Affiliations: From the Clinical Hospital of Chengdu Brain Science Institute, MOE Key Laboratory for Neuroinformation, University of Electronic Science and Technology of China, Chengdu, China (Zhao, Zhou, Zhou, Fu, Kendrick, Becker); the Department of Psychiatry and Division of Medical Psychology, University of Bonn, Bonn, Germany (Zimmermann, Dernbach, Scheele, Hurlemann); the Center for Economics and Neuroscience, Department of Epileptology, University of Bonn, Bonn, Germany (Weber); the Department of NeuroCognition, Life and Brain Center, Bonn, Germany (Weber); and the Institute of Medical Psychology, Center for Psychosocial Medicine, University Hospital, Heidelberg University, Heidelberg, Germany (Eckstein).

Funding: This work was supported by the National Natural Science Foundation of China (NSFC, 91632117; 31530032), the Fundamental Research Funds for the Central Universities of China (ZYGX2015Z002), the Sichuan Science and Technology Department (2018JY0001) and the German Research Foundation (DFG, grant: BE5465/2-1, HU1302/4-1).

\section{Competing interests: None declared.}

Contributors: K. Zimmermann, D. Scheele, B.Weber, R. Hurlemann, K. Kendrick and B. Becker designed the study. K. Zimmermann, C. Dernbach and M. Eckstein acquired the data, which W. Zhao, X. Zhou, F. Zhou and M. Fu analyzed. W. Zhao, D. Scheele and B. Becker wrote the article, which all authors reviewed. All authors approved the final version to be published and can certify that no other individuals not listed as authors have made substantial contributions to the paper.

\section{References}

1. Koob GF, Volkow ND. Neurobiology of addiction: a neurocircuitry analysis. Lancet Psychiatry 2016;3:760-73.

2. Koob GF, Volkow ND. Neurocircuitry of addiction. Neuropsychopharmacology 2010;35:217-38. 
3. UNODC. World drug report 2018. New York: United Nations Publications; 2018.

4. Curran HV, Freeman TP, Mokrysz C, et al. Keep off the grass? Cannabis, cognition and addiction. Nat Rev Neurosci 2016;17:293-306.

5. Volkow ND, Swanson JM, Evins AE, et al. Effects of cannabis use on human behavior, including cognition, motivation, and psychosis: a review. JAMA Psychiatry 2016;73:292-7.

6. Wrege J, Schmidt A, Walter A, et al. Effects of cannabis on impulsivity: a systematic review of neuroimaging findings. Curr Pharm Des 2014;20:2126-37.

7. Bossong MG, Kahn RS. The salience of reward. JAMA Psychiatry 2016;73:777-8

8. Becker B, Wagner D, Gouzoulis-Mayfrank E, et al. Altered parahippocampal functioning in cannabis users is related to the frequency of use. Psychopharmacology (Berl) 2010;209:361-74.

9. Wagner D, Becker B, Gouzoulis-Mayfrank E, et al. Interactions between specific parameters of cannabis use and verbal memory. Prog Neuropsychopharmacol Biol Psychiatry 2010;34:871-6.

10. Lazarus RS, Folkman S. Coping and adaptation. In: Gentry WD editor. The Handbook of Behavioral Medicine. New York: Guilford, 1984: 282-325.

11. Sinha R. The role of stress in addiction relapse. Curr Psychiatry Rep 2007;9:388-95.

12. Sinha R. Chronic stress, drug use, and vulnerability to addiction. Ann N Y Acad Sci 2008;1141:105-30.

13. Cheetham A, Allen NB, Yücel M, et al. The role of affective dysregulation in drug addiction. Clin Psychol Rev 2010;30:621-34.

14. Quinn PD, Fromme K. Self-regulation as a protective factor against risky drinking and sexual behavior. Psychol Addict Behav 2010;24:376-85.

15. Hyman SM, Sinha R. Stress-related factors in cannabis use and misuse: implications for prevention and treatment. J Subst Abuse Treat 2009;36:400-13.

16. Li CR, Sinha R. Inhibitory control and emotional stress regulation: neuroimaging evidence for frontal-limbic dysfunction in psychostimulant addiction. Neurosci Biobehav Rev 2008;32:581-97.

17. van Leeuwen JMC, Vink M, Fernández G, et al. At-risk individuals display altered brain activity following stress. Neuropsychopharmacology 2018;43:1954-60.

18. Wesley MJ, Lile JA, Hanlon CA, et al. Abnormal medial prefrontal cortex activity in heavy cannabis users during conscious emotional evaluation. Psychopharmacology (Berl) 2016;233:1035-44.

19. Zimmermann K, Yao S, Heinz M, et al. Altered orbitofrontal activity and dorsal striatal connectivity during emotion processing in dependent marijuana users after 28 days of abstinence. Psychopharmacology (Berl) 2018;235:849-59.

20. Zimmermann K, Walz C, Derckx RT, et al. Emotion regulation deficits in regular marijuana users. Hum Brain Mapp 2017;38:4270-9.

21. Simons J, Correia CJ, Carey KB. A comparison of motives for marijuana and alcohol use among experienced users. Addict Behav 2000;25:153-60.

22. Schlossarek S, Kempkensteffen J, Reimer J, et al. Psychosocial determinants of cannabis dependence: a systematic review of the literature. Eur Addict Res 2016;22:131-44.

23. Sheehan DV, Lecrubier $\mathrm{Y}$, Sheehan $\mathrm{KH}$, et al. The Mini International Neuropsychiatric Interview (MINI): the development and validation of a structured diagnostic psychiatric interview for DSM-IV and ICD-10. J Clin Psychiatry 1998;59:22-33.

24. Kogler L, Gur RC, Derntl B. Sex differences in cognitive regulation of psychosocial achievement stress: brain and behavior. Hum Brain Mapp 2015;36:1028-42.

25. Potenza MN, Hong KA, Lacadie CM, et al. Neural correlates of stress-induced and cue-induced drug craving: influences of sex and cocaine dependence. Am J Psychiatry 2012;169:406-14.

26. Chung KC, Peisen F, Kogler L, et al. The influence of menstrual cycle and androstadienone on female stress reactions: an fMRI study. Front Hum Neurosci 2016;10:44.
27. Eckstein M, Scheele D, Weber K, et al. Oxytocin facilitates the sensation of social stress. Hum Brain Mapp 2014;35:4741-50.

28. Jager G, Block RI, Luijten M, et al. Tentative evidence for striatal hyperactivity in adolescent cannabis-using boys: a cross-sectional multicenter fMRI study. J Psychoactive Drugs 2013;45:156-67.

29. Zhou F, Zimmermann K, Fei X, et al. Shifted balance of dorsal versus ventral striatal communication with frontal reward and regulatory regions in cannabis dependence. Hum Brain Mapp 2018; 39:5062-73.

30. Beck AT, Steer RA, Brown GK. Manual for the Beck Depression Inventory-II. San Antonio (TX): Psychological Corporation; 1996

31. Roese NJ, Jamieson DW. Twenty years of bogus pipeline research: a critical review and meta-analysis. Psychol Bull 1993;114:363-75.

32. Filbey FM, Dunlop J. Differential reward network functional connectivity in cannabis dependent and non-dependent users. Drug Alcohol Depend 2014;140:101-11.

33. Spielberger CD, Gorsuch RL, Lushene RE. Manual for the state-trait anxiety inventory. Palo Alto (CA): Consulting Psychologists Press; 1970.

34. Watson D, Clark LA, Tellegen A. Development and validation of brief measures of positive and negative affect: the PANAS scales. J Pers Soc Psychol 1988;54:1063-70.

35. Brickenkamp R. d2 Aufmerksamkeits-Belastungstest. Gottingen, Germany: Hogrefe; 2002

36. Agrawal A, Budney AJ, Lynskey MT. The co-occurring use and misuse of cannabis and tobacco: a review. Addiction 2012;107:1221-33.

37. Becker B, Hurlemann R. Unraveling the role of the amygdala in nicotine addiction. In: Preedy V, editor. Neuropathology of drug addictions and substance misuse. Cambridge (MA): Academic Press; 2016: 272-81.

38. Franklin TR, Jagannathan K, Hager N, et al. Brain substrates of early (4h) cigarette abstinence: identification of treatment targets. Drug Alcohol Depend 2018;182:78-85.

39. Dedovic K, Renwick R, Mahani NK, et al. The Montreal Imaging Stress Task: using functional imaging to investigate the effects of perceiving and processing psychosocial stress in the human brain. J Psychiatry Neurosci 2005;30:319-25.

40. Maldjian JA, Laurienti PJ, Kraft RA, et al. An automated method for neuroanatomic and cytoarchitectonic atlas-based interrogation of fMRI data sets. Neuroimage 2003;19:1233-9.

41. Brett M, Anton J-L, Valabregue R, et al. Region of interest analysis using an SPM toolbox [abstract]. Presented at the 8th International Conference on Functional Mapping of the Human Brain; 2002 Jun. 2-6; Sendai, Japan.

42. McLaren DG, Ries ML, Xu G, et al. A generalized form of contextdependent psychophysiological interactions (gPPI): a comparison to standard approaches. Neuroimage 2012;61:1277-86.

43. Dedovic K, Rexroth M, Wolff E, et al. Neural correlates of processing stressful information: an event-related fMRI study. Brain Res 2009;1293:49-60.

44. Breese GR, Sinha R, Heilig M. Chronic alcohol neuroadaptation and stress contribute to susceptibility for alcohol craving and relapse. Pharmacol Ther 2011;129:149-71.

45. Wardle MC, Munafò MR, De Wit H. Effect of social stress during acute nicotine abstinence. Psychopharmacology (Berl) 2011;218:39-48.

46. Ming $Q$, Zhong $X$, Zhang $X$, et al. State-independent and dependent neural responses to psychosocial stress in current and remitted depression. Am J Psychiatry 2017;174:971-9.

47. McRae-Clark AL, Carter RE, Price KL, et al. Stress-and cue-elicited craving and reactivity in marijuana-dependent individuals. Psychopharmacology (Berl) 2011;218:49-58.

48. Gilman JM, Curran MT, Calderon V, et al. Altered neural processing to social exclusion in young adult marijuana users. Biol Psychiatry 2016;1:152-9.

49. Menon V, Rivera SM, White CD, et al. Dissociating prefrontal and parietal cortex activation during arithmetic processing. Neuroimage 2000;12:357-65. 
50. Schurz M, Radua J, Aichhorn M, et al. Fractionating theory of mind: a meta-analysis of functional brain imaging studies. Neurosci Biobehav Rev 2014;42:9-34.

51. Zhang S, Li C-SR. Task-related, low-frequency task-residual, and resting state activity in the default mode network brain regions. Front Psychol 2012;3:172.

52. Zhang S, Li C-SR. A neural measure of behavioral engagement: task-residual low-frequency blood oxygenation level-dependent activity in the precuneus. Neuroimage 2010;49:1911-8.

53. Filbey FM, Schacht JP, Myers US, et al. Marijuana craving in the brain. Proc Natl Acad Sci U S A 2009;106:13016-21.

54. De Bellis MD, Wang L, Bergman SR, et al. Neural mechanisms of risky decision-making and reward response in adolescent onset cannabis use disorder. Drug Alcohol Depend 2013;133:134-45.

55. Aloi J, Blair KS, Crum KI, et al. Adolescents show differential dysfunctions related to alcohol and cannabis use disorder severity in emotion and executive attention neuro-circuitries. Neuroimage Clin 2018;19:782-92.

56. Roser P, Lissek S, Tegenthoff M, et al. Alterations of theory of mind network activation in chronic cannabis users. Schizophr Res 2012;139:19-26.

57. Hu S, Ide JS, Zhang S, et al. The right superior frontal gyrus and individual variation in proactive control of impulsive response. $J$ Neurosci 2016;36:12688-96.

58. Etkin A, Büchel C, Gross JJ. The neural bases of emotion regulation. Nat Rev Neurosci 2015;16:693-700.

59. Zhang S, Li CR. Functional connectivity mapping of the human precuneus by resting state fMRI. Neuroimage 2012;59:3548-62.

60. Becker B, Wagner D, Gouzoulis-Mayfrank E, et al. The impact of early-onset cannabis use on functional brain correlates of working memory. Prog Neuropsychopharmacol Biol Psychiatry 2010;34: 837-45.

61. Weinstein A, Livny A, Weizman A. Brain imaging studies on the cognitive, pharmacological and neurobiological effects of cannabis in humans: evidence from studies of adult users. Curr Pharm Des 2016;22:6366-79.

62. Kanayama G, Rogowska J, Pope HG, et al. Spatial working memory in heavy cannabis users: a functional magnetic resonance imaging study. Psychopharmacology (Berl) 2004;176:239-47.

63. Fransson P, Marrelec G. The precuneus/posterior cingulate cortex plays a pivotal role in the default mode network: evidence from a partial correlation network analysis. Neuroimage 2008; 42:1178-84.

64. Raichle ME, MacLeod AM, Snyder AZ, et al. A default mode of brain function. Proc Natl Acad Sci U S A 2001;98:676-82.

65. Buckner RL, Andrews-Hanna JR, Schacter DL. The brain's default network: anatomy, function, and relevance to disease. Ann NY Acad Sci 2008;1124:1-38

66. Livny A, Cohen $\mathrm{K}$, Tik N, et al. The effects of synthetic cannabinoids (SCs) on brain structure and function. Eur Neuropsychopharmacol 2018;28:1047-57.

67. Gilman JM, Schuster RM, Curran MT, et al. Neural mechanisms of sensitivity to peer information in young adult cannabis users. Cogn Affect Behav Neurosci 2016;16:646-61.

68. Daumann J, Hensen G, Thimm B, et al. Self-reported psychopathological symptoms in recreational ecstasy (MDMA) users are mainly associated with regular cannabis use: further evidence from a combined cross-sectional/longitudinal investigation. Psychopharmacology (Berl) 2004;173:398-404.

\section{$J P N^{\prime}$ 's top viewed articles*}

1. Why is depression more prevalent in women? Albert

J Psychiatry Neurosci 2015;40(4):219-21

2. N-Acetylcysteine in psychiatry: current therapeutic evidence and potential mechanisms of action Dean et al.

J Psychiatry Neurosci 2011;36(2):78-86

3. Mental illness is like any other illness: a critical examination of the statement and its impact on patient care and society

Malla et al.

J Psychiatry Neurosci 2015;40(3):147-50

4. Inappropriate benzodiazepine use in elderly patients and its reduction

Tannenbaum

J Psychiatry Neurosci 2015;40(3):E27-8

5. Adolescence as a unique developmental period Jaworska and MacQueen J Psychiatry Neurosci 2015;40(5):291-3

6. Treatment of ADHD in patients with bipolar disorder

Girard and Joober

J Psychiatry Neurosci 2017;42(6):E11-12

7. Legalizing marijuana

Leyton

J Psychiatry Neurosci 2016;41(2):75-6

8. Mindfullness-based treatments for posttraumatic stress disorder: a review of the treatment literature and neurobiological evidence

Boyd et al.

J Psychiatry Neurosci 2018;43(1):7-25

*Based on page views on PubMed Central of content published in the last 10 years.

Updated February 3, 2020 